\title{
Edad de la menopausia y su relación con la menarquia, gestaciones, hábito de fumar y uso de anticonceptivos en el IMI y HSJD de Santafé de Bogotá
}

\author{
Jaime Rojas C.*; William Onatra $\mathrm{H}^{* *}$; Waine Montoya G.***
}

RESUMEN OBJETIVO: Establecer la relación entre la edad de la menopausia, menarquia, número de gestaciones, hábito de fumar y uso de anticonceptivos orales.

MATERIAL Y METODOS: Se realizó una encuesta a 100 mujeres postmenopáusicas hospitalizadas del servicio de ginecología del IMI y 65 de la consulta externa del HSJD de Bogotá escogidas al azar. De los 165 casos se investigó la edad de la menopausia, número de gestaciones y el estudio de anatomía patológica. En 65 casos de la consulta externa el antecedente de uso de cigarrillo y anticonceptivos orales, la evaluación estadística incluye la prueba de chicuadrado y la razón de disparidad.

RESULTADOS: La edad de la menopausia para este grupo fue de 49,6 \pm 4.5 años, la menarquia $13.8 \pm 1.42$ años. Estadísticamente no hubo correlación entre la edad de la menopausia frente a la menarquia, número de gestaciones, hábito de fumar o uso de anticonceptivo oral.

CONCLUSION: Se describe el promedio de edad para este grupo hospitalario de mujeres postmenopáusicas no encontrándose relación con la menarquia, número de gestaciones y uso de cigarrillo o anovulatorios.

PALABRAS CLAVES:.Menopausia, menarquia, gestaciones, cigarrillo, anovulatorios.

SUMMARY OBJECTIVE: To know if the age of menopause have any relation with the age of menarche, number of pregnancies, smoke habits and the use of anticonceptive treatment in postmenopausic women.

MATERIAL AND METHODS: 100 postmenopausie women were screening by random in the ginecology service of the IMI and 65 from the HSJD external service of Bogota in this $\mathbf{1 6 5}$ cases was analized the age of menopause, the for hospitalization; patologic anatomy, number of pregnancies; and in $\mathbf{6 5}$ cases the use of cigarrettes and anticonceptives, the statistic analises included the "chi 2 " probe and the dispair ration.

RESULTS: The menopause age in this group of women were $49.6 \pm 4.5$ years, the age of the menarche were $13.8 \pm 1.4$ years.

Bey statistic there were no relation between the menopause, menarche, numbers of pregnancies, smoke habits and use of and anticonceptives.

CONCLUSION: After analized the average age of this hospitalarian group of post menopausic women there were no relation with the age of the first menarche, numbers of pregnancies and the use of cigarrettes and oral anticonceptives.

KEY WORDS: Menopause, menarche, pregnancies, cigarrettes, anticonceptives.

\section{Introducción}

Estudios realizados en el Hospital San Juan de Dios de Cúcuta (10) muestran que la edad promedio de la menopausia fue de 47 años, el número promedio de embarazos fue de 8 con una tasa de $9.4 \%$ de abortos y menopausia precoz (< de 40 años) en el $9.3 \%$ de sus casos, algunos autores han considerado la menopausia precoz por debajo de los 30 años (2).

* Especialista en Ginecología y Obstetricia. Universidad Nacional de Colombia.

** Profesor Asistente. Departamento de Obstetricia y Ginecología. Facultad de Medicina de la Universidad Nacional de Colombia.

*** Estadístico Facultad de Matemáticas. Universidad Nacional de Colombia.
En Estados Unidos, Escocia, Estocolmo y Suecia, la edad promedio es de $50 \pm 1.5$ años en el tiempo usual para ocurrencia del último período menstrual $(3,8,14)$. El uso de terapia estrogénica de reemplazo durante el período perimenopáusico demora la aparición de la menopausia en más o menos dos años (17).

En USA la pubertad está ocurriendo entre $9.1 \mathrm{a} \pm 17.7$ años con un promedio de la menarquia de 12.8 años (16) y se sabe, que la edad de la pubertad ha disminuido 1 a 3 meses en los últimos 175 años.

La edad de la menarquia ha sido considerada como un predictor potencial para la edad de la menopausia. Algunos estụdios no muestran ninguna relación entre estos dos parámetros (9). Estado marital, paridad y 
estado socioeconómico no han mostrado asociación con alguna tendencia en la edad de la menopausia (19). La paridad ha demostrado que aquellas mujeres que tuvieron 5 o más niños, la edad de la menopausia se presentó un año más tarde (19). El uso del cigarrillo se asocia con menopausia espontánea más temprana (9).

Los trastornos del ciclo son el primer signo del climaterio, aunque algunas mujeres premenopáusicas refieren oleadas de calor como primer signo. Los cambios en la función menstrual siguen varios patrones: 1) La mayoría de las mujeres presentan una disminución gradual de la cantidad y duración de la menstruación hasta la amenorrea (15). 2) Una minoría de pacientes tendrá sangrado premenstrual y un aumento en la frecuencia y duración del sangrado debido a la disminución de los progestágenos y de un aumento en la producción periférica de estrógenos que en algunas ocasiones lleva a la hiperplasia 0 al CA endometrial (17).

El diagnóstico exacto de la aparición de la menopausia no es fácil, excepto en los casos de ablación quirúrgica. Después de un año de amenorrea se ha considerado como el límite, aunque la mujer que recibe anticonceptivos o terapia de suplencia hormonal puede enmascarar la verdadera edad de la menopausia (13).

El cuadro clínico de alteraciones del ciclo, inestabilidad vasomotora, trastornos del comportamiento como irritabilidad y depresión con elevaciones de los niveles de FSH por encima de 100 UI/MI., son diagnósticas del inicio de este período. La histeroscopia permite determinar características de la cavidad uterina que se vuelve pequeña, lisa con escaso tejido endometrial, hemorragias petequiales y la aparición de una banda fibrótica blanca transversal localizada en el fondo uterino (15). El estudio del endometrio puede mostrar inicialmente proliferación y posteriormente una capa delgada compuesta de glándulas de estrato basal, dilatación quística (14). Toda hemorragia o el aumento mayor de $3 \mathrm{~mm}$. a nivel endometrial por ecografía debe ser llevado a legrado o biopsia que muestra una precisión diagnóstica entre 76 y $97 \%$ (19).

\section{Materiales y métodos}

Se tomaron 100 historias clínicas al azar de mujeres postmenopáusicas entre 40 y 60 años que ingresaron al servicio quirúrgico de Ginecología del Instituto Materno Infantil (IMI) y se encuestaron además 65 mujeres de las mismas características que asistían de la consulta externa del Hospital San Juan de Dios de la ciudad de Santafé de Bogotá entre meses de febrero a noviembre de 1990.

Se investiga la edad de la menopausia, menarquia, número de gestaciones y a 65 de ellas uso o no de cigarrillo o anticonceptivos orales durante su vida reproductiva.

Se agruparon en 3 categorías de acuerdo con la edad de la aparición de la menopausia. Grupo I. = menopausia temprana antes de los 44 años. Grupo II menopausia normal 44-53 años y grupo III menopausia tardía después de los 54 años. En la edad de la menarquia se conformaron también tres grupos: Grupo I temprana, menor de 12 años; Grupo II normal, de 12-15 años; y grupo III tardía, mayor de 16 años. En relación con el tabaquismo, se incluyeron las que fumaban un paquete al día por más de 5 años y las que usaron anticonceptivos orales o inyectables por más de 5 años. Estadísticamente se usó la prueba de chi-cuadrado y la razón de disparidad.

\section{Resultados}

La distribución de frecuencia de la menopausia se muestra en la tabla 1.

Tabla 1

\section{DISTRIBUCION DE EDAD DE LA MENOPAUSIA POR GRUPOS DE EDAD}

\begin{tabular}{|l|r|r|r|}
\hline Grupos de edad & $\mathbf{n}$ & \multicolumn{1}{|c|}{$\%$} & \\
\hline $40-41$ & 8 & 4.8 & \\
$42-43$ & 11 & 6.7 & \\
$44-45$ & 21 & 12.7 & \\
$46-47$ & 17 & 10.3 & \\
$48-49$ & 30 & 18.2 & Promedio 48.6 \\
& & & DS 4.5 \\
$50-51$ & 38 & 23.1 & \\
$52-53$ & 19 & 11.5 & \\
$54-55$ & 12 & 7.3 & \\
$56-57$ & 7 & 4.2 & \\
$58-59$ & 165 & 100.0 & \\
& & \\
\hline
\end{tabular}

La edad promedio de la menopausia para las 165 pacientes fue de 48.6 años con DS 4.5 . Utilizando lo anterior y para efectos de establecer relaciones, se construyeron los intervalos para la menopausia y la menarquia de tal manera que el $68.3 \%$ de los valores fueron incluidos.

De la misma manera como se establecieron unos intervalos para la edad de la menopausia, se hizo para la edad de la menarquia. Es así como se tomó la edad promedio de la menarquia, $13.8 \pm 1.42$; se construyó un intervalo que compara el $68.3 \%$ de las edades, con un límite inferior de 12 y un límite superior de 15 .

Establecidos los parámetros anteriores se procedió a establecer las siguientes hipótesis: Ho: La edad de la menopausia es independiente de la edad de la menarquia. Ha: La edad de la menopausia y la edad de la menarquia están relacionadas.

A un nivel de confianza del $95 \%$ se acepta la hipótesis nula, con un chi cuadrado X2 $=2.893$, grados de libertad $(G L)=4$ y una $P=0.576$ demostrando que la edad de la menopausia es independiente de la edad de la menarquia. Cuadro 1.

Cuadro 1

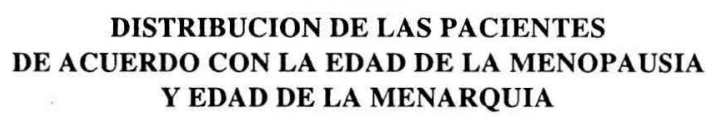

\begin{tabular}{|c|c|c|c|c|}
\hline $\begin{array}{l}\text { Edad } \\
\text { menopausia }\end{array}$ & \multicolumn{4}{|c|}{$\begin{array}{l}\text { Edad } \\
\text { menarquia } \\
\qquad \text { Años }\end{array}$} \\
\hline & $12<$ & $12-15$ & $>16$ & Total \\
\hline & $\mathrm{n} \%$ & $\mathrm{n} \%$ & $\mathrm{n} \%$ & $\mathrm{n} \%$ \\
\hline 44 & 10.6 & 1710.3 & 10.6 & 1911.5 \\
\hline $44-53$ & 53.0 & 10764.8 & 137.8 & 12575.7 \\
\hline 546 más & 0 & 1710.3 & 42.5 & 2112.7 \\
\hline Total & 63.6 & 14185.4 & 1810.9 & 165100 \\
\hline
\end{tabular}

$\mathrm{x}=13.8 \pm 1.42 \quad \mathrm{X} 2=2.893 \quad \mathrm{GL}=4 \quad \mathrm{P}=0.576$ 
Para las 2 variables anteriores (edad de la menopausia y edad de la menarquia), se intentó construir un modelo donde la edad de la menarquia fuera la variable independiente y la edad de la menopausia, la variable dependiente; si denotamos a X como la edad de la menarquia y a Y como la edad de la menopausia, los modelos construidos son: Modelo lineal $\mathrm{Y}=47,97+0.19 \mathrm{X}$ coeficiente de correlación $\mathrm{r}=$ 0.01601 . Modelo logarítmico $\mathrm{Y}=46.75+0.71$ In X. Modelo exponencial $4,1829 \mathrm{X} \mathrm{Y}=45.67$ y modelo potencial 0.057 $\mathrm{Y}=41.67 \mathrm{X}$, coeficiente de correlación $\mathrm{r}=0,0626$. Como puede observarse, el coeficiente de correlación es muy pequeño, lo cual nos indica que no hay correlación entre las 2 variables; confirmando la edad de la menopausia, es independiente de la edad de la menarquia.

La paridad promedio para las 165 pacientes fue de 6.2 con DS 4.1. El mayor número de casos, $64.89 \%$ corresponde a las grandes multíparas mayores de 5 partos, siguiéndole en frecuencia las multíparas, $21.2 \%$, primigestantes, $7.29 \%$ y multíparas, $6.69 \%$.

Con el fin de establecer una relación entre la edad de la menopausia y la paridad, se usó la prueba estadística $\mathrm{Ch}$ cuadrado $\mathrm{X} 2=9.193$ con $6 \mathrm{GL}$ y P de $=0.163$. Con estos datos se acepta la hipótesis nula donde la edad de la menopausia es independiente de la paridad, a pesar de existir en forma porcentual una tendencia de mayor edad de menopausia a mayor número de partos. Cuadro 2 .

\section{Cuadro 2}

\section{DISTRIBUCION DE LAS PACIENTES DE ACUERDO CON LA EDAD DE LA MENOPAUSIA Y PARIDAD}

\begin{tabular}{|l|ll|lll|ll|ll|l|l|l|}
\hline Menopausia & \multicolumn{7}{|c|}{ Paridad } & \multicolumn{2}{|l|}{ Total } \\
\hline Años & Go & & \multicolumn{2}{|c|}{ G1 } & & \multicolumn{2}{|c|}{ G2-4 } & \multicolumn{2}{|l|}{ G5 } & \multicolumn{2}{|l|}{ Tot } \\
\hline & n & $\%$ & n & $\%$ & n & $\%$ & n & $\%$ & n & $\%$ \\
\hline$<44$ & 1 & 0.6 & 4 & 2.4 & 2 & 1.2 & 12 & 7.2 & 19 & 11.5 \\
\hline $4-53$ & 10 & 6.0 & 7 & 4.2 & 29 & 17.6 & 79 & 47.8 & 125 & 75.7 \\
\hline$>54$ & 0 & & 1 & 0.6 & 4 & 2.4 & 16 & 9.7 & 21 & 12.8 \\
\hline Total & 11 & 6.6 & 12 & 7.2 & 35 & 21.2 & 107 & 64.8 & 165 & 100 \\
\hline
\end{tabular}

$\mathrm{X}=6.2 \pm 4.1 \mathrm{X} 2=9.193 \mathrm{GL}=6 \mathrm{P}=0.163$

En las 65 encuestas se investiga si la edad de la menopausia está influenciada sobre el hecho de fumar o no planteándose las siguientes hipótesis: Ho: La edad de la menopausia es independiente del hecho de fumar o no. Ha: La edad de la menopausia y el hecho de fumar están relacionados. Las pruebas de significancia mostraron un X2 $=1.1$ con 2 grados de libertad y $\mathrm{P}=0.571$ aceptando para este grupo que la edad de la menopausia es independiente del hecho de fumar para este grupo etáreo. Cuadro 3.

Con el intervalo propuesto para la edad de la menopausia y el uso o no de anovulatorios, se trató de establecer una relación y se plantearon las siguientes hipótesis. Ho: La edad de la menopausia es independiente del uso de anovulatorios orales. Ha: La edad de la menopausia y el uso de anovulatorios están relacionados.

Se hizo la prueba estadística correspondiente a un nivel de confianza del $95 \%$, con un X2 $=1.69,2$ grados de libertad y $\mathrm{P}=0.248$ confirmando para este grupo que la edad de la menopausia es independiente del uso de anovulatorios. Cuadro 4
Cuadro 3

DISTRIBUCION DE LAS PACIENTES DE ACUERDO CON LA EDAD DE LA MENOPAUSIA Y EL HABITO DE FUMAR

\begin{tabular}{|c|cc|cc|lc|}
\hline \multirow{2}{*}{ Edad } & \multicolumn{5}{|c|}{ Hábito de fumar } & \multicolumn{2}{c|}{} \\
\hline Menopausia & \multicolumn{2}{|c|}{$\mathrm{Si}$} & \multicolumn{2}{c|}{ No } & \multicolumn{2}{c|}{ Total } \\
\hline Años & $\mathrm{n}$ & $\%$ & $\mathrm{n}$ & $\%$ & $\mathrm{n}$ & $\%$ \\
\hline$<44$ & 2 & 3.9 & 8 & 12.3 & 10 & 15.4 \\
\hline $44-53$ & 7 & 10.7 & 43 & 66.2 & 50 & 76.9 \\
\hline$>54$ & 0 & & 5 & 7.7 & 5 & 7.7 \\
\hline Total & 9 & 13.8 & 56 & 86.2 & 65 & 100 \\
\hline
\end{tabular}

$\mathrm{X} 2=1.122 \mathrm{GL}=2 \mathrm{P}=0.571$

Cuadro 4

DISTRIBUCION DE LAS PACIENTES

DE ACUERDO CON LA EDAD DE MENOPAUSIA Y USO O NO DE ANOVULATORIOS

\begin{tabular}{|c|cc|lc|l|l|}
\hline Edad & \multicolumn{5}{|c|}{ Anovulatorios } & \multicolumn{2}{c|}{} \\
\hline Menopausia & \multicolumn{2}{|c|}{ Si } & \multicolumn{2}{|c|}{ No } & \multicolumn{2}{c|}{ Total } \\
\hline Años & $\mathrm{n}$ & $\%$ & $\mathrm{n}$ & $\%$ & $\mathrm{n}$ & $\%$ \\
\hline$<44$ & 1 & 1.6 & 9 & 13.8 & 10 & 5.4 \\
\hline $44-53$ & 10 & 15.4 & 40 & 61.5 & 50 & 76.9 \\
\hline$>54$ & \multicolumn{2}{|c|}{0} & 5 & 7.7 & 5 & 7.7 \\
\hline Total & 11 & 13.817. & 54 & 83.0 & 65 & 100 \\
\hline
\end{tabular}

$\mathrm{X} 2=1.696 \mathrm{GL}=2 \mathrm{P}=0.428$

En el estudio de anatomía patológica fueron reportadas 62 muestras de las cuales el $75 \%$ correspondió al endometrio artrófico, proliferativo $9 \%$ y adenocarcinoma de endometrio $8 \%$ (tabla 2).

Tabla 2

ANATOMIA PATOLOGICA ENDOMETRIAL DE 62 PACIENTES POSTMENOPAUSICAS HOSPITALIZADAS EN EL SER'VICIO DE GINECOLOGIA DEL I.M.I.

\begin{tabular}{|l|c|c|}
\cline { 2 - 3 } \multicolumn{1}{c|}{} & M & $\%$ \\
\hline Endometrio atrófico & 45 & 75.8 \\
\hline Endometrio proliferativo & 12 & 9 \\
\hline Adenocarcinoma de endometrio & 4 & 8 \\
\hline $\begin{array}{l}\text { Hiperplasia adenomatosa Vs } \\
\text { Endometrio proliferativo con } \\
\text { focos de hiperplasia quística }\end{array}$ & 2 & 3.2 \\
\hline Pólipo endometrial & 1 & 1.6 \\
\hline Material no apto para Dx & 1 & 6.6 \\
\hline
\end{tabular}




\section{Discusión}

La edad promedio de la menopausia ha permanecido estable alrededor de los 50 años desde el siglo VI D.C. (21), con algunas variaciones según país, raza y condiciones socioeconómicas. En Norteamérica es de 51.4, Noruega 49.7 y Nueva Guinea en el Africa 43.6 años (22). En la siguiente (tabla 3) se pueden observar estas diferencias (23).

Tabla 3

EDAD DE LA MENOPAUSIA SEGUN PAIS Y RAZA

\begin{tabular}{|c|c|c|c|c|}
\hline País & Año & Autor & Raza & P. Edad \\
\hline \multirow[t]{2}{*}{ Inglaterra } & 1964 & Fromer & Blanca & 49.8 \\
\hline & 72 & Mckinley & Blanca & 50.7 \\
\hline Alemania & 1972 & Hofmann & Blanca & 49.6 \\
\hline Suecia & 1979 & Lindguist & Blanca & 51.2 \\
\hline \multirow[t]{2}{*}{ Estados Unidos } & 1974 & Treloar (16) & Blanca & 49.8 \\
\hline & 1966 & McMahon & Negra & 49.3 \\
\hline \multirow[t]{3}{*}{ Sudáfrica } & 1960 & Abrahamson & Blanca & 48.1 \\
\hline & & & Negra & 47.7 \\
\hline & & Benjamín & Blanca & 48.7 \\
\hline Escocia & 1973 & Thompson & Blanca & 50.1 \\
\hline Suiza & 1961 & Hauser. & Blanca & 49.8 \\
\hline India & 1966 & Wyon & Asiática & 44.0 \\
\hline Nueva Guinea & 1973 & Gray & Asiática & 43.6 \\
\hline Grecia & 1979 & Batrinos & Blanca & 46.6 \\
\hline \multirow[t]{2}{*}{ España } & 1977 & Botella & Blanca & 50.1 \\
\hline & 1985 & Caballero & Blanca & 48.6 \\
\hline \multirow[t]{2}{*}{ Colombia } & 1970 & Luzardo & Mestiza & 47.0 \\
\hline & 1990 & $\begin{array}{c}\text { Presidente } \\
\text { estudio }\end{array}$ & Mestiza & 48.6 \\
\hline
\end{tabular}

Por los datos anteriores podemos aceptar que la edad de la menopausia para este grupo etáreo se encuentra dentro del promedio mundial.

La edad de la menarquia fue 13.8 años dentro del promedio teniendo en cuenta que un estudio americano (16) y otros hechos en Colombia es de 12.8 - 13 años, años (20-24). Su relación con la edad de la menopausia ha creado controversia y es así como han postulado 2 hipótesis: por un lado la menarquia temprana, menopausia temprana y por otro la menarquia tardía menopausia tardía, pero las investigaciones realizadas demuestran se encuentra que la edad de la menopausia se mantiene independiente de la menarquia $(4,8)$.

La relación de la menopausia tardía y número de gestaciones ha sido observado en el pasado en algunas de nuestras comunidades con promedio de más de 10 hijos entre los 45 y 55 años. El presente estudio demuestra estadísticamente que no existe relación entre el número de gestaciones y la edad de la menopausia aunque se observa una ligera tendencia en gestantes mayores de 5 partos a presentar su menopausia alrededor de los 52 años (9.7\%), datos sugeridos por Edwars en Inglaterra (25).

Es sabido que el cigarrillo en las pacientes postmenopáusicas aumenta el riesgo de accidentes cerebro vascular e infarto de una relación de 2 a 1 entre las fumadoras de más de 35 cigarrillos al día (29). Estudios transversales tanto europeos como americanos han demostrado que la edad de la menopausia se adelanta $1.5-2.0$ años en las fumadoras de más de 35 cigarrillos al día con un promedio de 50.4 años frente a las no fumadoras cuyo promedio es de 52.4 años (30). Tabla 4. El número tan pequeño de casos en este estudio no permite establecer diferencias y solo podemos aceptar que sólo el $10 \%$ de las pacientes postmenopáusicas han fumado más de un paquete de cigarrillos al día.

Tabla 4

EDAD DE LA MENOPAUSIA Y USO DE CIGARRILLO (30)

\begin{tabular}{|c|c|c|c|}
\hline Referencia & & No. Fumadores & Fumadores \\
\hline$(29)$ & Stampfer & 52.4 & 50.4 \\
\hline$(30)$ & Andersen & 49.9 & 47.9 \\
\hline
\end{tabular}

Diferentes autores han postulado que el uso de anticonceptivos retrasan la menopausia $(31,32)$ y son útiles en prevención del cáncer de ovario y de endometrio (33). El presente trabajo pretendía sólo conocer si el uso de anticonceptivos influía sobre la edad de la menopausia. Estadísticamente no hay diferencias pero dado el número tan pequeño es imposible confirmar esta hipótesis.

El endometrio durante el período premenopáusico fisiológicamente debe ser proliferativo, sin embargo en diferentes estudios sobre hemorragia uterina disfuncional el estado del endometrio es variable, encontrándose E. secretor $30-40 \%$, E. proliferativo 50-60\% y atrófico según el grupo de edad del 10-20\% (10, 26-27). Las cifras del presente trabajo están dentro de los límites reportados en la literatura $(12,16,19,28)$. 


\section{BIBLIOGRAFIA}

1. Barlon J., Emerson K., SAXENA. Estradiol production after ovariotomy for carcinoma of the breast. N. England J. Med. 1969; 28: 633-637.

2. Dates. G W on the nature of the hot flash Clin. Obstet. Gynecol 1981; 24 : 231-341.

3. Bengtsson C., Linoquist O., Repvall $\mathrm{L}$. Is the menopausal age rapidly ohanging. Maturitas. 1979; 1: 159-164.

4. Benson RC. Current obstetric and gynecologic diagnosis and treatment 2th edition, Lange Medical Publications 1989.

5. Burnet FM. Inmunological approach to aging. Lancet 1970; 2: 358.

6. Diccionario Terminológico de Ciencias Médicas; undécima edición Salvat 1974.

7. Gribb JJ. Histeroscopy as and aid gynecologic diagnosis Obstet Gynecol 1960; 15: 401-593.

8. Jazman LJB. Epidemiology of climateric and postclimateric complaints. Ncrm Res 1973; 2: 22-34.

9. Jick H., Porter J., Morrinson AS. Relation between smoking and age of natural menopause report from the Boston University Medical Center. Lancet 1977; 1: 8026: 1355-1357.

10. Luzardo M., F. Pérez G. Climaterio y menopausia Rev. Col. Obstet. y Ginecol. 1970; 21: 273-315.

11. Mcmahon B., Vorester J. National Center for health statistics. Age at menopause, U.S. $1500^{\circ}-1962$ Washington D.C. USPHS Publication 1000. 1966; 11, 19.

12. Perrill JA. Tratamiento de la hemorragia postmenopáusica. Clin. Obstet. Ginecol. 1981; 1: 287-301.

13. Nohmcnovici C. How doss one confirm the definitive character of the menopausal on a clinical basis. Rev. Fren. Gynecol. Obstet. 1985; 4: 183186.

14. Robbins Stanley: Patología estructural y funcional. Ed. Interamericana $1974 ; 1186$.

15. Thonpson B., Hart SA., Duvia D. Menopausal age and synptomatology in general practice J. Biosoc Jol. 1973; 5: 71-82.

16. Treolar AC. Menarche, menopause and intervining fecundability Hum. Biol. 1974; 16: 89-98.

17. Treolar AC. Mestrual oyclinicity and the premenopause Maturitas 1981; 3: $249-269$.
18. Myshak G. Menopause in mothers of multiple births and mothers of singletons only. J. Biol. 1978; 25: 52-61.

19. Cutler W.B. The medical manegemento of menopause and premenopause J. B. Lippin Colt. 1984.

20. Jubis A. et al. Motivos de consulta más frecuentes en 620 adolescentes. Rev. Col. Obstet. Ginecol. 1972; 23: 399-409.

21. Utian WH. Menopause in modern perspective. A guide to clinical practice. New York. Apleton Century Crofts. 1980.

22. Gray RH. The menopause epidemiological and demographic considerations. In the menopause. Beard RJ edit. MTP press Lancaster. 1976; 25-40.

23. Botella Llusia J. La edad crítica. Epidemiología del climaterio. Edit. Salvat. Barcelona, España. 1990; 51-61.

24. Jaramillo R. Adolescencia, Rev. Col. Obstet. Ginecol. 1984; 35: 401424.

25. Edwards RQ. The menopause. Research in reproduction. 1978; 10: 13.

26. Rybo G., Leam J., Tibbin E. Epidemiology of menstrual blood loss. In Baird DT, Michie EA. Mechanism of menstrual bleeding. Raven Press 1985; 181-193.

27. Sánchez J., Onatra W. Hemorragia uterina disfuncional en el IMI 1987 1988, Rev. Col. Obstet. Ginecol. 1990; 41: 247-252.

28. Richard Kustan Ch., Kase N. Diagnosis and management of perimenoppausal and postmenopausal bleeding. Clin. Obstet. Gynecol. 1992; 14: 1: 169-185.

29. Stampfer MJ., Colditz GR. Willeti W. Menopause and heart disease. Ann New York Academy Scienc 1990; 592: 193-203.

30. Andersen FS., Diers CJ. Is cigarette smoking promotor of menopause Act. Med. Scand. 1982; 212: 137-139.

31. Kinley MS., Jefferies M. An investigation on the age of the menopause, J. Biol. Sci. 1972; 4: 161-168.

32. Jazmann LJB., Van Lith ND. The age of the menopause in the Nettherlands. Int. J. Fertil. 1969; 14: 106-117.

33. Mc Gonigle KF., Huggisn GR. Oral contraceptives and breast disease Fertil Steril 1991; 56: 799-819. 